\title{
IS INVASIVE PRESSURE MONITORING MORE RELIABLE THAN NON- INVASIVE IN PATIENTS WITH CARDIOVASCULAR PATHOLOGY? \\ A CASE REPORT
}

\author{
Fernandes $A^{1}$, Colmbra L ${ }^{1}$, Delgado I $^{1}$, Carvalho J ${ }^{1}$, Bentes $\mathrm{C}^{1}$ \\ ${ }^{1}$ DePARTMENT OF ANAESTHESIOLOGY, CENTRO Hospitalar VILA NOVA DE GAIA/ESPINHO, EPE
}

\section{BACKGROUND}

Patients undergoing carotid endarterectomy (CEA) require strict arterial blood pressure (BP) control to maintain adequate cerebral perfusion. Invasive blood pressure (IBP) is the gold standard, however artifacts may lead to erroneous readings. ${ }^{1}$

We report a case of CEA using IBP monitoring.

\section{CASE REPORT}

\section{IDENTIFICATION}

Male, 64 years old, ASA III

$\checkmark$ Diffuse atheromathosis, dyslipidemia and non-medicated hypertension

$\checkmark$ Diagnosis: carotid stenosis

$\checkmark$ Surgery: elective right carotid endarterectomy

$\checkmark$ Anesthesia technique: General Anesthesia (GA)

\section{MONITORIZATION}

- ASA standard, neuromuscular block monitoring, anesthesia depth and cerebral oximetry

\section{- PREANAESTHETIC ASSESSMENT NONINVASIVE BP} (NIBP)

- no significant difference between right and left arms $(180 / 90 \mathrm{mmHg})$

- IBP monitoring was placed in left radial artery after several attempts

- INTRAOPERATIVE

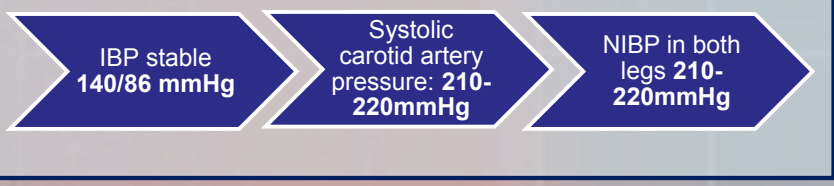

NIBP was used to monitor and guide the BP target until the end of the procedure. Surgery proceeded uneventfully.

\section{POSTOPERATIVE}

NIBP was used to monitor and guide the BP target at PACU, despite the presence of IBP monitoring

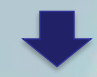

POSTOPERATIVE DAY 2:

At PACU: NIBP shows Hypertensive Crisis

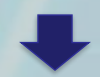

Cervical Neck Haematoma

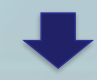

Emergent Surgery under GA.

Intraoperatively the BP was assessed with NIBP

POSTOPERATIVE DAY 8 :

Patient was discharged without further complications.

\section{Discussion}

IBP allow beat-by-beat measures with optimization of BP in order to improve cerebral perfusion during CEA. NIBP may be an alternative, however is not continuous and is expected to be less accurate than the IBP. ${ }^{1}$ The high IBP-NIBP difference (>40 $\mathrm{mmHg}$ ) was clinically relevant and in this patient might be explained by diffuse atheromatosis. NIBP was compatible with carotid systolic pressure, indicating that, in this case was a reliable and accurate method of monitoring.

\section{LeARning Points}

IBP can be inaccurate in patients with diffuse atheromatosis. This pathology may have clinical relevance and may demand non-invasive arterial pressure measurement may help during and after surgery avoiding inappropriate treatments. 